\title{
CONSERVAÇÃO E QUALIDADE DE MIRTILO ORGÂNICO UTILIZANDO REVESTIMENTO COMESTÍVEL A BASE DE FÉCULA DE MANDIOCA
}

\section{CONSERVATION AND QUALITY OF ORGANIC RABITTEYE BLUEBERRY (Vaccinium ashei Reade) USING CASSAVA STARCH EDIBLE COATING}

\author{
${ }^{1}$ Marcibela Stülp, ${ }^{2}$ Edmar Clemente, ${ }^{3}$ Dalany Menezes Oliveira, ${ }^{4 \Xi}$ Bruna Barbara Bucalão Gnas \\ ${ }^{1 \bowtie, 2,3,4}$ Universidade Estadual de Maringá - UEM - Maringá - Brasil- marcibelastulp@ gmail.com; ( ${ }^{\bowtie}$ Autor \\ correspondente)
}

\begin{abstract}
Resumo
Um dos grandes desafios da produção de mirtilo (Vaccinium ashei, Reade) no sistema orgânico consiste em prolongar a vida pós-colheita dos frutos diminuindo as perdas e prolongar a oferta do produto no mercado nacional e internacional. $O$ uso de revestimentos comestíveis é uma técnica que vem sendo utilizada em frutos de forma eficiente para o prolongamento da vida de prateleira. Desta forma o objetivo deste trabalho foi avaliar o efeito do revestimento comestivel a base de fécula de mandioca na conservação e qualidade de mirtilo orgânico produzido no município de Palmas no Estado do Paraná. Os frutos de mirtilo, cultivar Clímax, receberam a aplicação do revestimento comestível a base de fécula de mandioca e foram armazenados a $10^{\circ} \mathrm{C}$ e 85 a $90 \%$ de UR., e as avaliações realizadas no tempo zero, 7 e 14 dias de armazenamento. Observou-se que o tratamento filme comestivel a base de fécula de mandioca não apresentou alterações para as variáveis analisadas na temperatura e período de armazenamento utilizado podendo, ser considerado um tratamento eficiente na conservação de mirtilo, mantendo as características químicas dos frutos para comercialização.
\end{abstract}

Palavras-chave: mirtilo; revestimento comestível; conservação.

\section{Introdução}

O mirtilo (Vaccinium ashey, Reade), também conhecido como blueberry ou arándano é uma frutífera que pertence à família Ericaceae, e está classificado dentro da subfamília Vaccinioideae, na qual se encontra o gênero Vaccinium (TREHANE, 2004; DONADIO et al., 1998). O mirtilo é uma planta nativa da América do Norte, encontrada desde o Sul dos Estados Unidos até o Leste do Canadá. A importância desta planta está em seu fruto, que é uma baga, com um sabor doce excelente, muito apreciado (JARA-AYALA, 1999). 
Os frutos podem ser utilizados para consumo "in natura", sucos geléias, iogurtes, entre outros, porém a oferta ainda é muito pequena, o que o torna um fruto desconhecido pela grande maioria da população brasileira, inclusive pela população do Sul do país, que é a região mais recomendada para o seu cultivo (DONADIO et al., 1998). O mirtileiro produz frutos com diâmetro entre 8 e $22 \mathrm{~mm}$, de sabor agridoce (CHILDERS e LYRENE, 2006), com diversas propriedades nutracêuticas e alto potencial antioxidante, em razão da presença de compostos fenólicos (KALT et al., 2007).

O mirtilo apresenta grande importância comercial, especialmente nos Estados Unidos (BRAZELTON e STRIK, 2007) e em alguns países da Europa, como Itália e Alemanha (STRIK, 2005). No Brasil, a produção de pequenas frutas como o mirtilo, ainda são restritas a poucas áreas, mas as perspectivas de cultivo são promissoras (ANTUNES, 2005).

O cultivo comercial do mirtilo está em franca expansão em países da América do Sul como Chile, Argentina e Uruguai, com área de produção de aproximadamente 6.500 ha. A expansão da cultura nesses países é influenciada, em grande parte, pela demanda da entressafra de países do hemisfério norte como os Estados Unidos (STRIK, 2005; BRAZELTON e STRIK, 2007). Essas demandas de mercado podem gerar oportunidades de negócio para o setor produtivo brasileiro, desde que haja adoção de tecnologia para a produção e a utilização de cultivares adequados (ANTUNES e MADAIL, 2005).

No Brasil, os principais cultivares pertencem ao grupo rabitteye (ANTUNES e RASEIRA, 2006). Apresentam como características: elevado vigor, plantas longevas, alta produtividade, tolerância ao calor e à seca, baixa exigência na estação fria, floração precoce, longo período entre floração e maturação e frutos firmes, com longa vida pós-colheita se conservados adequadamente (EHLENFELDT et al., 2007).

Um fator importante para o aumento das áreas cultivadas com mirtilo é o crescente interesse pelos antioxidantes naturais de extratos de plantas devido à sua baixa toxicidade em relação aos antioxidantes sintéticos. Extratos de frutas, vegetais, cereais e seus subprodutos industriais são ricos em antioxidantes, em ácido ascórbico, tocoferóis, carotenóides e em compostos fenólicos e têm demonstrado eficaz atividade antioxidante em sistemas modelos (WOLFE et al., 2003; MANACH et al., 2004).

Os compostos fenólicos são formados no metabolismo secundário dos vegetais possuem funções de defesa contra o ataque de pragas. Já em animais e humanos tem-se observado que são capazes de reagir com radicais livres formando radicais estáveis. Esse poder de neutralização das estruturas radiculares dos compostos fenólicos é devido à sua estrutura química formada por pelo menos um anel aromático com grupamentos hidroxilas (GIADA e MANCINI-FILHO, 2006). 
Além da sua atividade antioxidante direta, pesquisas recentes têm destacado múltiplas funções e mecanismos importantes relacionados à habilidade dos compostos fenólicos de se ligarem a receptores celulares e transportadores de membranas e influenciarem a expressão gênica, a sinalização e a adesão celular (MANACH et al., 2005).

Os "alimentos orgânicos" incluem todos os produtos alimentícios produzidos com a adoção de técnicas e com o uso de normas da agricultura orgânica, atendendo a critérios específicos na embalagem, armazenamento e transporte, preservando ao máximo seus atributos de qualidade (CHITARRA e CHITARRA, 2005).

As práticas de manuseio pós-colheita são tão importantes quanto às práticas culturais. E muitos problemas relacionados com a perda acentuada de qualidade e deterioração dos alimentos são o resultado de danos sucessivos e cumulativos que estes sofrem durante todos os seus períodos de manuseio, armazenamento e transporte (CORTEZ et al., 2002).

A utilização de películas comestíveis tem sido bastante explorada para revestimento de frutas e hortaliças frescas, visando minimizar a perda de umidade e reduzir as taxas de respiração, além de conferir aparência brilhante e atraente (AZEREDO, 2003). O uso de películas com esse propósito constitui vantagem econômica, evitando a necessidade de estocagem em atmosfera controlada que implicaria em custos operacionais e de utilização de equipamentos. A função a ser desempenhada pelo filme depende do produto alimentício e principalmente do tipo de deterioração a que este produto está submetido (MAIA et al., 2000).

A função de uma cobertura comestível é atuar como uma barreira à perda de umidade, controlar a respiração do fruto e evitar contaminações microbiológicas e químicas. A atmosfera modificada criada pelo revestimento gera um aprisionamento físico do $\mathrm{CO}_{2}$ dentro do fruto. Se a permeação de oxigênio $\left(\mathrm{O}_{2}\right)$ para seu interior é reduzida, acorrerá prolongamento do tempo de maturação. Além disso, os revestimentos comestíveis têm a vantagem da biodegradabilidade que os torna "ambientalmente correto" (ASSIS e LEONI, 2003).

$\mathrm{O}$ uso dos revestimentos também serve como adjunto para proporcionar economia com materiais de embalagem, manutenção da qualidade, prolongamento da vida útil dos frutos, aumentando o seu período de comercialização. Desta forma, o objetivo deste trabalho foi avaliar a influência do tratamento a base de fécula de mandioca associada ao armazenamento refrigerado na conservação de mirtilo, avaliando a qualidade e o poder antioxidante de frutos de mirtilo produzido em sistema orgânico de produção no município de Palmas sul do Estado do Paraná. 


\section{Material e métodos}

Os frutos de mirtilo (Vaccinium ashei, Reade), cultivar Clímax, utilizados no experimento foram produzidos em sistema orgânico de cultivo na safra de 2009/2010, no município de Palmas no Estado do Paraná. A colheita foi realizada quando os frutos atingiram coloração azulada em 100\% da superfície da casca, sendo acondicionados em caixas plásticas sem perfurações, tipo PET (tereftalato de polietileno) transparentes com tampa e transportados ao Laboratório de Bioquímica de Alimentos, da Universidade Estadual de Maringá.

Após a chegada ao laboratório os frutos foram sanitizados selecionados e em seguida receberam a aplicação do revestimento comestível a base de fécula de mandioca. Após a secagem os frutos foram novamente acondicionadas em caixas plásticas sem perfurações, tipo PET transparentes e com tampa com capacidade de 150g, sendo levados para o armazenamento refrigerado a $10{ }^{\circ} \mathrm{C}$ e 85 a $90 \%$ de UR., e as avaliações realizadas no tempo zero, 7 e 14 dias de armazenamento. Os frutos que não receberam nenhuma cobertura de revestimento foram embalados e armazenados nas mesmas condições dos demais frutos revestidos.

No final de cada período de armazenamento foram realizadas as seguintes análises: avaliação da perda de massa das frutas, determinada por diferença entre a massa inicial e a massa final após o período de armazenamento, com auxílio de balança semi-analítica; teor de solúveis totais (SST), expressos em ${ }^{\circ}$ Brix, obtido em refratômetro digital; acidez total titulável (ATT), determinada por titulometria de neutralização com $\mathrm{NaOH}$ 0,1 M expressa em porcentagem de ácido cítrico (AOAC, 1992); pH determinado por meio de potenciômetro digital; relação SST/ATT (ratio); determinação de açúcares totais pelo método de Lane-Eynon segundo os Métodos físicoquímicos para análises de alimentos do Instituto Adolfo Lutz (2005); compostos fenólicos totais e antocianinas totais.

O teor de compostos fenólicos totais foi determinado pelo método espectrofotométrico de Folin-Ciocalteau utilizando ácido gálico como padrão de referência. A leitura foi realizada a 765 $\mathrm{nm}$ em espectrofotômetro. Os extratos de mirtilo formados por $2,5 \mathrm{~g}$ de mirtilo mais $50 \mathrm{~mL}$ de álcool $50 \%$, foram centrifugados por 5 minutos e uma alíquota de $0,2 \mathrm{~mL}$ de amostra diluente foi transferida para um tubo com tampa de rosca e lhe foram adicionados $1,8 \mathrm{~mL}$ de água destilada e 10 $\mathrm{mL}$ do reagente Folin-Ciocalteau (Sigma Aldrich Chemical Co.) diluídos em água destilada 1:10. Esses reagentes ficaram em repouso de 3 a 8 min e a eles foram adicionados $8 \mathrm{~mL}$ de carbonato de sódio a 7,5\%. Os tubos foram deixados em repouso por $2 \mathrm{~h}$ ao abrigo da luz e, na sequência, feita a leitura da absorbância a $740 \mathrm{~nm}$. Um reagente branco foi conduzido nas mesmas condições. Foi 
construída uma curva analítica contendo 100, 80,60,40, 20, $10 \mu \mathrm{g} \cdot \mathrm{mL}^{-1}$ de ácido gálico e os resultados expressos em mg de ácido gálico $\mathrm{g}^{-1}$ de mirtilo (SINGLETON e ROSSI, 1965).

Para extração das antocianinas, as frutas foram trituradas, homogeneizadas e uma alíquota de $10 \mathrm{~g}$ pesada e adicionada de solução extratora em pH 2,0 (etanol $96^{\circ} \mathrm{GL}+\mathrm{HCL} 1 \mathrm{M}$ ) em Becker com capacidade de $125 \mathrm{~mL}$. As amostras foram mantidas no escuro sob refrigeração $\left(\sim 4^{\circ} \mathrm{C}\right)$ durante 16 horas, quando foram filtradas (filtro Watchman $\mathrm{N}^{\circ} 1$ ) e lavadas com a solução extratora até completar o volume de $10 \mathrm{~mL}$ medidos em balão volumétrico.

Uma alíquota de $5 \mathrm{~mL}$ da solução filtrada foi adicionada a $20 \mathrm{~mL}$ de solução extratora e armazenada no escuro em temperatura ambiente durante 30 minutos para estabilização. A avaliação do teor de antocianinas totais foi realizada por método espectrofotométrico, de acordo com protocolo de Lees e Francis (1972), e o teor de antocianinas expresso em miligramas de antocianinas em $100 \mathrm{~g}$ de mirtilo.

O revestimento comestível a base de fécula de mandioca, na concentração de $2,5 \%$ foi obtido a partir de $50 \mathrm{~g}$ de fécula de mandioca para $2 \mathrm{~L}$ de água, que foi dissolvido e levado para aquecimento a $70^{\circ} \mathrm{C}$, com agitação constante da suspensão até a geleificação, seguido de repouso até o completo resfriamento onde recebeu a adição de $20 \mathrm{~mL}$ de glicerol sofrendo nova agitação com o auxilio de um mixer durante 2 minutos.

O delineamento utilizado foi o de blocos casualizados, com três repetições e os dados obtidos foram submetidos à análise de variância, e as médias, comparadas pelo teste e de Tukey a $5 \%$ de probabilidade. Os dados foram processados pelo programa estatístico SAS.

\section{Resultados e discussão}

Observando os resultados referentes a variável pH (Tabela1) observou-se que houve um incremento nos valores ao longo da primeira semana de armazenamento. Resultado semelhante foi observado por Garcia et al., (1998b) em morangos, que atribuíram este aumento de pH a degradação de ácidos orgânicos, como o ácido málico e o ácido ascórbico, que ocorre no processo natural de senescência do produto. Este comportamento foi seguido de uma queda nos valores de $\mathrm{pH}$, comportamento que pode ser atribuído ao consumo destes ácidos no processo de transpiração e respiração dos frutos.

Os teores de sólidos solúveis totais (SST), encontrados no experimento apesar de não apresentar diferenças significativas entre a testemunha e os frutos revestidos com fécula de mandioca estão de acordo com os teores encontrados na literatura que teor de sólidos solúveis totais (SST) no mirtilo varia de $7 \%$ nos frutos verdes a $15 \%$ ou mais nos frutos maduros. Os açúcares são 
dos principais componentes solúveis e representam cerca de $80 \%$ da matéria seca, sendo que a glucose e a frutose são os principais açúcares existentes no mirtilo e os seus teores influenciam o sabor (SOUZA et al., 2007).

Segundo Souza et al., ( 2007), a qualidade de mirtilo depende também do conteúdo em ácidos orgânicos, mas o cultivar estudado não apresentou diferenças entre tratamentos no conteúdo em ácido cítrico, variando entre 0,19 e 0,24 g ácido cítrico $100 \mathrm{~g}-1$ de mirtilo, ao longo do experimento (Tabela 1). No entanto observou-se um incremento nos teores de ácido cítrico nos primeiros sete dias de armazenamento tanto para a testemunha quanto para os frutos revestidos com fécula de mandioca, seguido de um decréscimo nos teores de ácido cítrico ao final dos 14 dias do experimento. De acordo com Chitarra e Chitarra, (2005), este decréscimo pode ser justificado devido ao consumo dos ácidos pelo processo de respiração dos frutos.

Tabela 1 - Média da composição química e perda de massa em frutos de mirtilo orgânico tratados com revestimento comestível a base de fécula de mandioca.

\begin{tabular}{|c|c|c|c|}
\hline \multirow{3}{*}{ Tratamento } & \multicolumn{3}{|c|}{ Períodos de Armazenamento (dias) } \\
\hline & T1 $1^{1}$ & T2 & T3 \\
\hline & \multicolumn{3}{|c|}{$\mathbf{p H}^{2}$} \\
\hline Testemunha & $2,72 \mathrm{Aa} \pm 0,01$ & $3,01 \mathrm{Ab} \pm 0,02$ & $2,78 \mathrm{Aa} \pm 0,02$ \\
\hline \multirow[t]{2}{*}{ Fécula } & $2,71 \mathrm{Aa} \pm 0,03$ & $3,07 \mathrm{Ab} \pm 0,04$ & $2,76 \mathrm{Aa} \pm 0,04$ \\
\hline & \multicolumn{3}{|c|}{ SST ( ${ }^{\mathbf{0}}$ Brix) } \\
\hline Testemunha & $13,10 \mathrm{Aa} \pm 1,56$ & $12,40 \mathrm{Aa} \pm 0,46$ & $12,43 \mathrm{Aa} \pm 0,67$ \\
\hline Fécula & $12,60 \mathrm{Aa} \pm 1,14$ & $13,00 \mathrm{Aa} \pm 0,20$ & $13,90 \mathrm{Aa} \pm 1,64$ \\
\hline \multicolumn{4}{|c|}{ ATT (mg de ác. cítrico $100 \mathrm{mg}^{-1}$ de mirtilo) } \\
\hline Testemunha & $0,20 \mathrm{Aa} \pm 0,01$ & $0,24 \mathrm{Ab} \pm 0,03$ & $0,20 \mathrm{Aab} \pm 0,00$ \\
\hline Fécula & $0,19 \mathrm{Aa} \pm 0,00$ & $0,23 \mathrm{Ab} \pm 0,01$ & $0,21 \mathrm{Aab} \pm 0,01$ \\
\hline \multicolumn{4}{|c|}{ Ratio (SST/ATT) } \\
\hline Testemunha & $65,17 \mathrm{Aa} \pm 4,83$ & $52,63 \mathrm{Aa} \pm 5,07$ & $60,87 \mathrm{Aa} \pm 3,44$ \\
\hline Fécula & $67,05 \mathrm{Aa} \pm 6,15$ & $56,68 \mathrm{Aa} \pm 3,12$ & $66,84 \mathrm{Aa} \pm 6,80$ \\
\hline \multicolumn{4}{|c|}{ Açúcares Totais (\%) } \\
\hline Testemunha & $24,80 \mathrm{Aa} \pm 1,15$ & $20,99 \mathrm{Aa} \pm 1,11$ & $36,54 \mathrm{Ab} \pm 2,63$ \\
\hline Fécula & $25,95 \mathrm{Aa} \pm 3,33$ & $14,56 \mathrm{Ab} \pm 4,56$ & $33,29 \mathrm{Aa} \pm 6,36$ \\
\hline \multicolumn{4}{|c|}{ Compostos Fenólicos (mg de ác. gálico $100 \mathrm{~g}^{-1}$ de mirtilo) } \\
\hline Testemunha & $40,09 \mathrm{Aa} \pm 4,01$ & $28,56 \mathrm{Aa} \pm 2,10$ & $43,82 \mathrm{Ab} \pm 4,99$ \\
\hline Fécula & $36,59 \mathrm{Aab} \pm 10,80$ & $42,67 \mathrm{Aa} \pm 2,54$ & $40,68 \mathrm{Ab} \pm 3,57$ \\
\hline \multicolumn{4}{|c|}{ Antocianinas Totais (mg de antocianina $100 \mathrm{~g}^{-1}$ de mirtilo) } \\
\hline Testemunha & $65,03 \mathrm{Aa} \pm 20,42$ & $75,16 \mathrm{Aa} \pm 6,31$ & $68,94 \mathrm{Aa} \pm 4,20$ \\
\hline Fécula & $51,90 \mathrm{Aa} \pm 11,73$ & $73,09 \mathrm{Aa} \pm 10,09$ & $60,78 \mathrm{Aa} \pm 9,52$ \\
\hline \multicolumn{4}{|c|}{ Perda de Massa (\%) } \\
\hline Testemunha & $0,00 \mathrm{Aa} \pm 0,00$ & $2,92 \mathrm{Ab} \pm 0,32$ & $5,57 \mathrm{Ac} \pm 0,04$ \\
\hline Fécula & $0,00 \mathrm{Aa} \pm 0,00$ & $2,97 \mathrm{Ab} \pm 0,75$ & $5,80 \mathrm{Ac} \pm 0,93$ \\
\hline
\end{tabular}

${ }^{1}$ Períodos de Armazenamento: T1: zero dias; T2: sete dias; T3:14 dias; ${ }^{2}$ Médias seguidas de mesma letra maiúscula na coluna e minúscula na linha, não diferem entre si pelo teste de Tukey, a $5 \%$ de probabilidade. $\mathrm{n}=$ número de repetições

Segundo Souza et al., (2007) os açúcares e os ácidos têm uma evolução inversa ao longo do amadurecimento, sendo a sua relação uma indicação do estado de maturação dos frutos, mas não necessariamente da sua qualidade gustativa, esta evolução inversa entre o aumento dos açúcares e a 
diminuição da acidez total deve-se a duas razões principais: hidrólise de polissacarídeos e a formação de açúcar como produto secundário da conversão dos ácidos orgânicos.

Por causa da importância do componente doce, e ácido na qualidade de mirtilos, a razão de açúcar/ácido é habitualmente determinada, e é considerada como índice de qualidade (CHITARRA e CHITARRA, 2005). De acordo com os valores obtidos, os cultivares do presente estudo apresentam uma razão adequada para uma qualidade gustativa aceitável, demonstrando que o revestimento a base de fécula de mandioca não interfere nas características de qualidade dos frutos, sendo uma alternativa viável a sua conservação.

Os teores de compostos fenólicos e açúcares totais também não apresentaram diferenças entre os tratamentos e intervalos de tempo utilizados. No entanto, houve um incremento nos teores de açúcar aos 14 dias e nos teores de compostos fenólicos com o passar dos dias. Segundo Chitarra e Chitarra, (2005), este incremento pode ter ocorrido devido à perda de massa dos frutos ter aumentado de forma linear ao logo do experimento, diminuindo assim o teor de água nos tecidos favorecendo o acúmulo de açúcares e a síntese de compostos fenólicos nos frutos de mirtilo mesmo em pós-colheita. Em relação ao conteúdo de antocianinas podemos observar que não foram encontradas diferenças entre a testemunha e o tratamento fécula de mandioca nos valores de concentração em antocianinas no período de armazenamento.

Foram encontradas diferenças nos valores de $\%$ de perda de massa ao logo do tempo, mas não foram observadas diferenças entre o tratamento testemunha e fécula de mandioca. As perdas de água por transpiração, principal causa de perda de peso, originam alterações morfológicas importantes que podem afetar a cor e a textura do fruto. Segundo Chitarra e Chitarra (2005), perdas na ordem de $3 \%$ a $6 \%$ são suficientes para acarretar um declínio na qualidade, causando o murchamento. No entanto os valores de perda de massa foram inferiores a 5\%, ao fim de 14 dias de armazenamento a $10^{\circ} \mathrm{C}$ e 85 a $90 \%$ de UR, mantendo ainda o seu valor comercial.

\section{Conclusão}

O tratamento filme comestível a base de fécula de mandioca não apresentou diferenças para as variáveis analisadas na temperatura e períodos de armazenamento utilizados podendo ser considerado, um tratamento eficiente na conservação de mirtilo, mantendo as características químicas dos frutos para comercialização.

\section{Agradecimentos}

Ao Conselho Nacional de Desenvolvimento Científico e Tecnológico - CNPq, pelo suporte financeiro. 


\begin{abstract}
One of the greatest challenges on the production of rabbiteye blueberry (Vaccinium ashei Reade) in an organic system consists of prolonging the post harvesting life of the fruits reducing its losses in order to extend the offer of the product in the national and international market. The use of edible coating is a technique that has been used in fruits in an efficient way to increase the shelf life. Thus, the objective of this work was to evaluate the effect of the cassava starch edible coating in the conservation and quality of organic blueberries produced in the municipal district of Palmas, Paraná State. The blueberry fruits, Climax cultivar, were coated with edible cassava starch and stored at $10^{\circ} \mathrm{C}$ and 85 to $90 \% \mathrm{RH}$. The evaluations were carried out at the zero time, 7 and 14 days of storage. It was observed that the treatment with the cassava starch edible coating did not cause any changes for the analyzed variables, in the temperature and period of used storage. So, it might be an efficient treatment to be considered in the blueberry conservation, maintaining the chemical characteristics of the fruits for its commercialization.
\end{abstract}

Key-words: blueberry; edible coating; conservation.

\title{
Referências
}

ANTUNES, L. E. C.; MADAIL, J. C. M. Mirtilo: que negócio é este? Jornal da Fruta, v. 13, p. 8, 2005.

ANTUNES, L. E. C.; RASEIRA, M. C. B. Cultivo do mirtilo (Vaccinium spp.). Pelotas: Embrapa Clima Temperado, 2006. 99p. (Embrapa Clima Temperado. Sistema de Produção, 8).

AZEREDO, H. M. C. de. Películas comestíveis em frutas conservadas por métodos combinados: potencial da aplicação. Boletim do CEPPA, v. 21, n. 2, 2003.

BRAZELTON, D.; STRIK, B. C. Perspective on the U.S. and global blueberry industry. Journal of American Pomological Society, v. 61, p. 144-147, 2007.

CHILDERS, N. F.; LYRENE, P. M. Blueberries for growers, gardeners, promoters. Florida: E. O. Painter Printing Company, 2006. 266p.

CHITARRA, M. I. F.; CHITARRA, A. B. Pós-colheita de frutos e hortaliças: fisiologia e manuseio. 2. ed. rev. e ampl. Lavras: UFLA, 2005.

DONADIO, L. C.; NACHTIGAL, J. C.; SACRAMENTO, C. K. Frutas exóticas. FCAV- UNESP. Jaboticabal, SP. 1998. 279p.

EHLENFELDT, M. K.; ROWLAND, L. J.; OGDEN, E. L.;VINYARD, B. T. Floral bud cold hardiness of Vaccinium ashei, $V$. constablaei, and hybrid derivatives and the potencial for producing Northern-adapted rabbiteye cultivars. HortScience, v. 42, p. 1131-1134, 2007.

GIADA, M. L. R.; MANCINI FILHO, J. Importância dos compostos fenólicos da dieta na promoção da saúde humana. Publicatio UEPG: Ciências Biológicas e da Saúde, v. 12, n. 4, p. 7-15, 2006.

GOUGH, R.E. The highbush blueberry and its management. Nova York: Haworth Press, 1994. 272p.

JARA AYALA, F. DE LA. Amora - Framboesa - Groselha - Kiwi - Mirtilo e sua comercialização. Cinco Continentes. Editora Ltda. Porto Alegre, RS. 1999. 57p.

MAIA, L. H.; PORTE, A.; SOUZA, V. F. de. Filmes comestíveis: aspectos gerais, propriedades de barreira à umidade e o oxigênio. Boletim do CEPPA,v. 18, n. 1, 2000.

MANACH, C. Polyphenols: food sources and bioavailability. American Journal of Clinical Nutrition, v. 79, n. 5, p. 727-747, 2004.

SOUZA, M. B.; CURADO, T.;VASCONCELLOS, F. N.E: TRIGO, M. J. Mirtilo - Qualidade pós-colheita. Alentejo, Portugal, Folhas de Divulgação AGRO 556 n.8, 2007.

STRIK, B. C. Blueberry: an expanding world crop. Chronica Horticulturae, v. 45, p. 7-12, 2005. 
STRIK, B.C. Horticultural practices of growing highbush blueberries in the ever-expanding U.S. and global scene. Journal of the American Pomological Society, v. 61, p. 148-150, 2007.

WOLFE, K.; WU, X.; LIU, R. H. Antioxidant activity of apple peels. Journal of Agricultural and Food Chemistry, v. 53, p. 609-614, 2003. http://dx.doi.org/10.1021/jf020782a

Submetido em 29 mar. 2011, Aceito para publicação em 21 mai. 2012. 